\title{
Introducing GOLDMine: A new galaxy database on the WEB
}

\author{
G. Gavazzi ${ }^{1}$, A. Boselli ${ }^{2}$, A. Donati ${ }^{1}$, P. Franzetti ${ }^{3,1}$, and M. Scodeggio ${ }^{3}$ \\ 1 Università degli Studi di Milano-Bicocca, Piazza delle scienze 3, 20126 Milano, Italy \\ e-mail: alessandro.donati@mib.infn.it \\ 2 Laboratoire d'Astrophysique de Marseille, Traverse du Siphon, 13376 Marseille Cedex 12, France \\ e-mail: alessandro.boselli@oamp. fr \\ 3 Istituto di Astrofisica Spaziale e Fisica Cosmica, CNR, via Bassini 15, 20133 Milano, Italy \\ e-mail: paolo@mi.iasf.cnr.it; marcos@mi.iasf.cnr.it
}

Received 15 October 2002 / Accepted 11 December 2002

\begin{abstract}
The new World Wide Web site "GOLDMine" (Galaxy On Line Database Milano Network) (http:// goldmine.mib.infn.it) contains a multiwavelength data-base of an optically selected sample of 3267 galaxies in the Virgo cluster and in the Coma Supercluster. It is designed for professional astronomers who wish to find data and images for these galaxies. Data gathered in 15 years of observational campaigns by the authors or taken from the literature include general parameters (catalogue names, celestial coordinates, morphological type, recessional velocity etc.), multiwavelength continuum photometry (total $U V, U, B, V, J, H, K$, FIR and radio magnitudes/flux densities), line photometry ( $\mathrm{HI}, \mathrm{H}_{2}, \mathrm{H} \alpha$ ), dynamical parameters (rotational velocity from the $\mathrm{HI}$ and $\mathrm{H} \alpha$ lines, velocity dispersion) and structural parameters (light concentration index, effective radius and brightness, asymptotic magnitude) in the optical ( $B$ and $V)$ and Near Infrared $(H$ or $K)$ bands.

Images include finding charts, optical ( $B$ and $V), \mathrm{H} \alpha$, Near Infrared ( $H$ and/or $K$ ) and true color RGB frames (when available). Radial light profiles obtained from the $B, V, H$ or $K$ band images are also available. Integrated optical spectra along with broad Spectral Energy Distributions (SED) from the $U V$ to the radio domain are given. All images can be obtained in JPG format, but the original (reduced) FITS images can be downloaded as well. The database will be updated regularly and will be extended to other local clusters and superclusters. Astronomers who wish to have their images included in GOLDMine are strongly encouraged to send us their material.
\end{abstract}

Key words. atlases - galaxies: general

\section{Introduction}

Galaxies come in many different forms and sizes, but they can be broadly divided into two main species: Spirals, with a flattened, disk-like shape, blue colors, much gas and dust, and a widespread star formation activity that results in the presence within them of many young stars, and Ellipticals, with a spheroidal shape, red colors, little or no gas and dust, and no star formation activity, thus containing exclusively old stars. While these differences are well documented, and have been extensively studied over the last 70 years, astronomers are not yet able to convincingly explain the origin of such diversity. In fact, one of today's major open issues in astronomy is to obtain a plausible reconstruction of the processes of galaxy formation and evolution. The task is of course enormously complicated by the sheer disparity between cosmological and human time-scales, that makes it impossible for astronomers to witness galaxy evolution directly. One possible way to overcome this problem is to take advantage of the "time machine"

Send offprint requests to: G. Gavazzi, e-mail: giuseppe.gavazzi@mib.infn.it effect provided by the finite speed of light. Observing today galaxies at different distances means observing them at different epochs in the history of the Universe, and thus with different ages. Unfortunately distances must be very large before age differences become significant, and therefore unveiling the evolution of galaxies with direct observations of very distant objects is becoming a reality only today, with the advent of the $10 \mathrm{~m}$ class telescopes. Galaxies at progressively larger look-back times (redshift), thus in a younger evolutionary stage, will be observed in the near future, back to a small fraction of the present age of the Universe. This will provide us with a sequence of "fossil" galaxies, eventually disclosing the secret of their evolution, much as fossil organisms guided paleontologists tracing back the evolution of species. However, 15 years ago, when we initiated this project, a direct observational approach to the problem was unconceivable, because of the lack of proper telescopes and instrumentation. The only available option was to attack the evolutionary enigma starting from the study of evolved systems, i.e. the nearby galaxies. This approach is based on the conviction that "adult" galaxies 
still preserve some memory of their past, and that adequate observations would eventually help to disclose it.

With this purpose in mind we started in 1985 an observational campaign aimed at providing the phenomenology of local galaxies in the widest possible frequency range. We took observations and collected data from the literature from the $2000 \AA(U V)$ range to the centimetric radio domain, spending a large effort in making the literature data as homogeneous as possible with our own data. GOLDMine is designed to provide access to this massive data-set on local galaxies through the World Wide Web. Numeric parameters as well as the (reduced) scientific FITS images can be downloaded.

\section{The sample selection}

GOLDMine is focused on two regions of the sky: the Virgo cluster and the Coma Supercluster ${ }^{1}$. Among rich clusters of galaxies, the Virgo cluster is the nearest to us (17 Mpc, Gavazzi et al. 1999b) (see Fig. 1). The Coma supercluster contains two rich clusters (Coma \& Abell 1367) corresponding to the density enhancements in Fig. 2. In addition, this region contains a filament of nearly isolated galaxies, the so-called "Great Wall" at a distance of approximately $90 \mathrm{Mpc}\left(7000 \mathrm{~km} \mathrm{~s}^{-1}\right.$, Gavazzi et al. 1999a) from us (see Fig. 2). The studied regions contain 3 rich clusters of galaxies as well as isolated objects, thus providing the ideal laboratory for a comparative analysis of galaxies in different environments.

As opposed to other more extensive galaxy data-bases (e.g. NED, LEDA) which include all objects with published parameters, galaxies in GOLDMine were selected with the criterion of optical completeness. All galaxies brighter than a threshold magnitude were selected in both areas. In the Coma supercluster all galaxies brighter than $m_{\mathrm{p}}=15.7$ were selected from the Catalogue of Galaxies and of Clusters of Galaxies (CGCG) by Zwicky et al. (1961-1968) ${ }^{2}$. The Virgo region contains all (2096) galaxies brighter than $m_{\mathrm{p}}=20.0$ from the Virgo Cluster Catalogue (VCC) by Binggeli et al. (1985) ${ }^{3}$. Obviously, due to the factor of 5 difference in distance between the Virgo and the Coma clusters, this selection limit results in dwarf galaxies from being included in our database only for the Virgo cluster. However, globally GOLDMine covers the whole range (4 orders of magnitude) of luminosities spanned by real galaxies.

Altogether GOLDMine contains 3267 galaxies, approximately $60 \%$ of which are of early-type (elliptical and S0) and the remaining $40 \%$ of late-type (spirals and irregulars).

Extensive campaigns were carried out to observe as many as possible of the 3267 target galaxies through all possible observational windows, a task that we have not yet completed. Moreover we collected data from the literature and we made

\footnotetext{
1 An additional 382 galaxies (with $m_{\mathrm{p}} \leq 15.7$ ) in the A262 (PerseusPisces) and Cancer clusters and in the Hercules supercluster (A2147, A2151, A2197, A2199) will be included shortly in GOLDMine.

2 The number of galaxies meeting the $m_{\mathrm{p}} \leq 15.7$ criterion is 1082 . However 45 galaxies listed as "pairs" in the CGCG were split into fainter $\left(m_{\mathrm{p}} \leq 17.0\right)$ components. The resulting Coma galaxies in GOLDMine are 1127.

344 additional Virgo galaxies with $m_{\mathrm{p}} \leq 14.5,12 \mathrm{~h}<\mathrm{RA}<13 \mathrm{~h}$, but outside the area covered by the VCC are included.
}

all possible effords to homogenize them with the ones obtained by us.

\section{Database fields}

The parameters listed in the GOLDMine database are divided into 5 categories: General, Continuum and Line photometry, Dynamical and Structural. All parameters are extensively described and referenced in the section "GOLDMine glossary". See also the section "GOLDMine tutorial" to learn about using the database.

The parameters can be obtained from GOLDMine in two ways:

1) by querying the database for an individual galaxy name, or

2) by a multiple query "by parameters". In this case all galaxies in a given range of photographic magnitude and morphological type can be selected. The query criteria include also the availability of continuum, line photometry, dynamical and structural parameters.

General parameters include: CGCG (Zwicky et al. 19611968), NGC (Dreyer 1888), IC (Dreyer 1895, 1908), VCC (Binggeli et al. 1985), UGC (Nilson 1973) designations; Celestial coordinates precessed to the year 2000; isophotal diameters; photographic magnitude; heliocentric recessional velocity; distance and morphological type.

Continuum photometry parameters consist of total magnitudes from aperture or CCD photometry, uncorrected for extinction either internal or due to our Galaxy. They include: $U V$ (2000 ̊); optical $(U, B, V)$; Near-IR $(J, H, K)$ magnitudes; Far-IR (IRAS 60 and $100 \mu \mathrm{m}$ ); radio continuum (1420 and $610 \mathrm{MHz}$ ) fluxes.

Line photometry parameters include: the atomic (HI) and molecular $\left(\mathrm{H}_{2}\right)$ hydrogen mass (the corresponding fluxes can be derived using the source distance); the $\mathrm{H} \alpha+[\mathrm{NII}]$ line equivalent width and flux.

Dynamical parameters include: the width of the HI line, with a quality flag; the width of the $\mathrm{H} \alpha$ line and the central velocity dispersion.

Structural parameters include the light concentration index (C31); the effective radius $R_{\mathrm{e}}$; the effective surface brightness $\mu_{\mathrm{e}}$; the total asymptotic magnitude. These quantities are given separately for the $H, V$ and $B$ bands.

\section{Imaging}

The novelty of GOLDMine is its image section, where images can be downloaded in JPG and FITS format. They can be accessed via the the queries "by name" or "by parameters". Images include:

- Finding Charts from the Digitized Palomar Sky Survey. Each selected galaxy appears at the center of the $10 \times$ $10 \operatorname{arcmin}^{2}$ finding chart. When multiple catalogued galaxies appear in the same finding chart, each of them can be selected by clicking on their names. In this case the database and image queries are re-directed to the selected galaxy.

- Broad band images obtained in the $B, V, H$ and $K$ bands. 


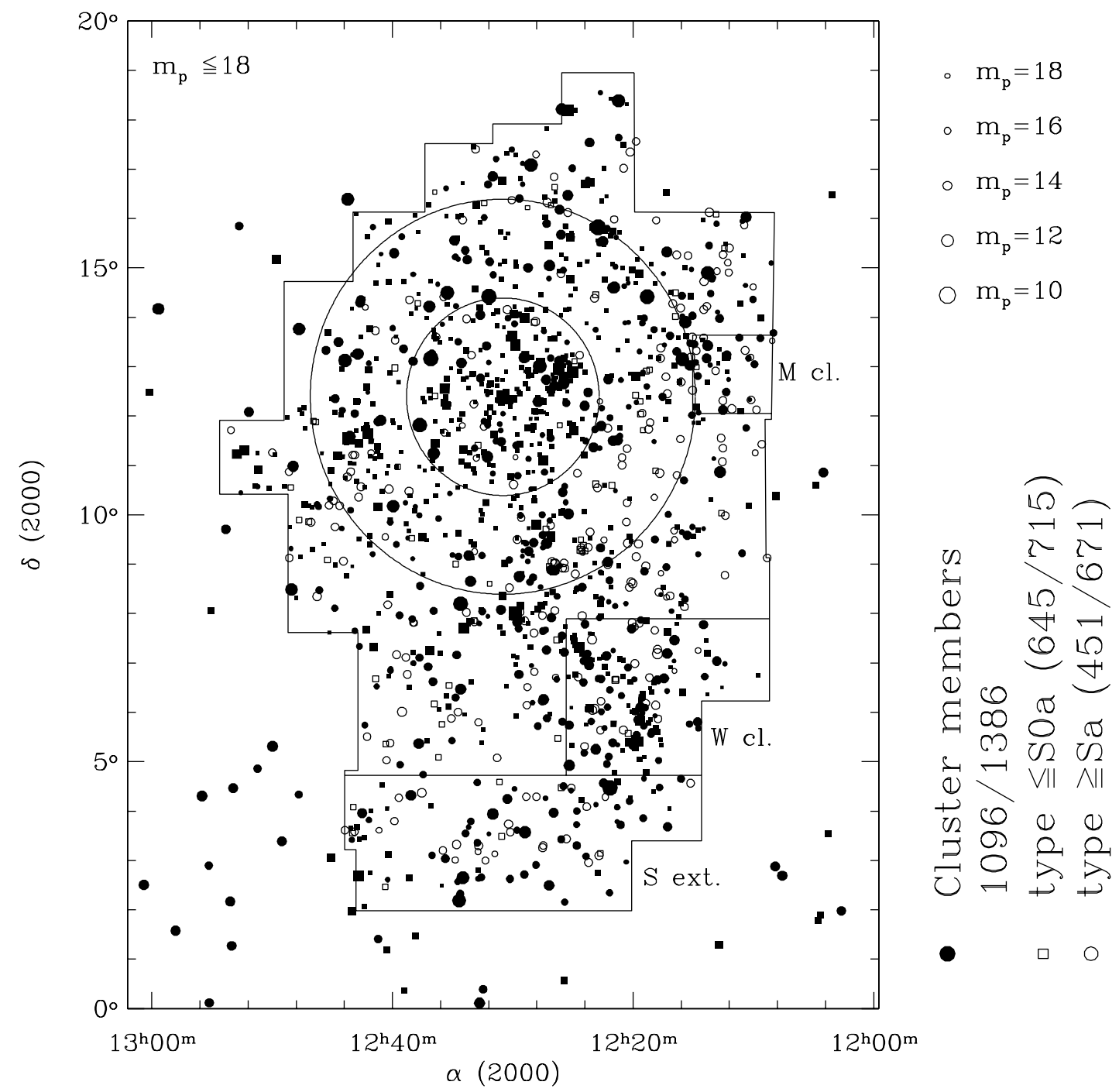

Fig. 1. Sky distribution of the 1386 galaxies brighter than $m_{\mathrm{p}} \leq 18.0$ in the Virgo cluster. Possible and spectroscopically confirmed cluster members (filled symbols) are 1096.

- Narrow band images in the light of $\mathrm{H} \alpha$. Each galaxy has two $\mathrm{H} \alpha$ images: the "OFF-band" one which gives the underlying stellar continuum near $\mathrm{H} \alpha$ and the "NET" one, obtained by subtracting the OFF-band image from the ONband one.

- RGB images. For few miscellaneous galaxies we combined several images to obtain "true" color pictures (only available in JPG format). Some are combinations of $H, V$ and $B$ frames, other of $\mathrm{H} \alpha$ OFF, ON and NET frames.

- Light profiles. Radial profiles of the light distribution as obtained on the available $(B, V, H)$ images. See Gavazzi et al. (2000). When at least two radial profiles are available the color radial profile is also shown (only available in JPG format).

- Spectroscopy. These are optical spectra integrated over the whole surface of the galaxy (obtained in drift-scan mode, i.e. by drifting the spectrograph slit over the galaxy extension), see Gavazzi et al. (2002). Spectra were obtained in several observational campaigns carried out with optical telescopes (OHP 1.9m, ESO 3.6m) by Gavazzi et al. (2002). Spectra exist for 141 galaxies, but 80 additional spectra will be included in GOLDMine shortly.

- SEDs. These are Spectral Energy Distributions from the $U V$ to the centimetric radio continuum obtained from broad-band photometry (only available in JPG format). The plotted data are total fluxes (extrapolated to the optical radii), unlike the individual aperture data given by NED. However they are given as observed, i.e. uncorrected for extinction from our Galaxy and for internal extinction ${ }^{4}$.

It is our goal to provide a homogeneous set of keywords in all FITS headers to characterize the data. These will include:

- Galaxy name.

- Effective integration time.

${ }^{4}$ some SEDs contain more data points than listed in the GOLDMine database. For example the IRAS $25 \mu \mathrm{m}$ flux and the fluxes obtained by ISO (CAM and PHOT) are plotted without being listed in GOLDMine. 


$$
\begin{aligned}
& \left(m_{p} \leqq \mathbb{1} \gamma\right) \\
& \text { - } \mathrm{m}_{\mathrm{p}}=17 \\
& \text { - } \mathrm{m}_{\mathrm{p}}=16 \\
& \text { - Supercluster members } \\
& \text { - } \mathrm{m}_{\mathrm{p}}=15 \\
& \text { ○ } \mathrm{m}_{\mathrm{p}}=14 \\
& \text { - } \quad \text { type } \leqq \mathrm{SOa}(387 / 595) \\
& \bigcirc \mathrm{m}_{\mathrm{p}}=13
\end{aligned}
$$

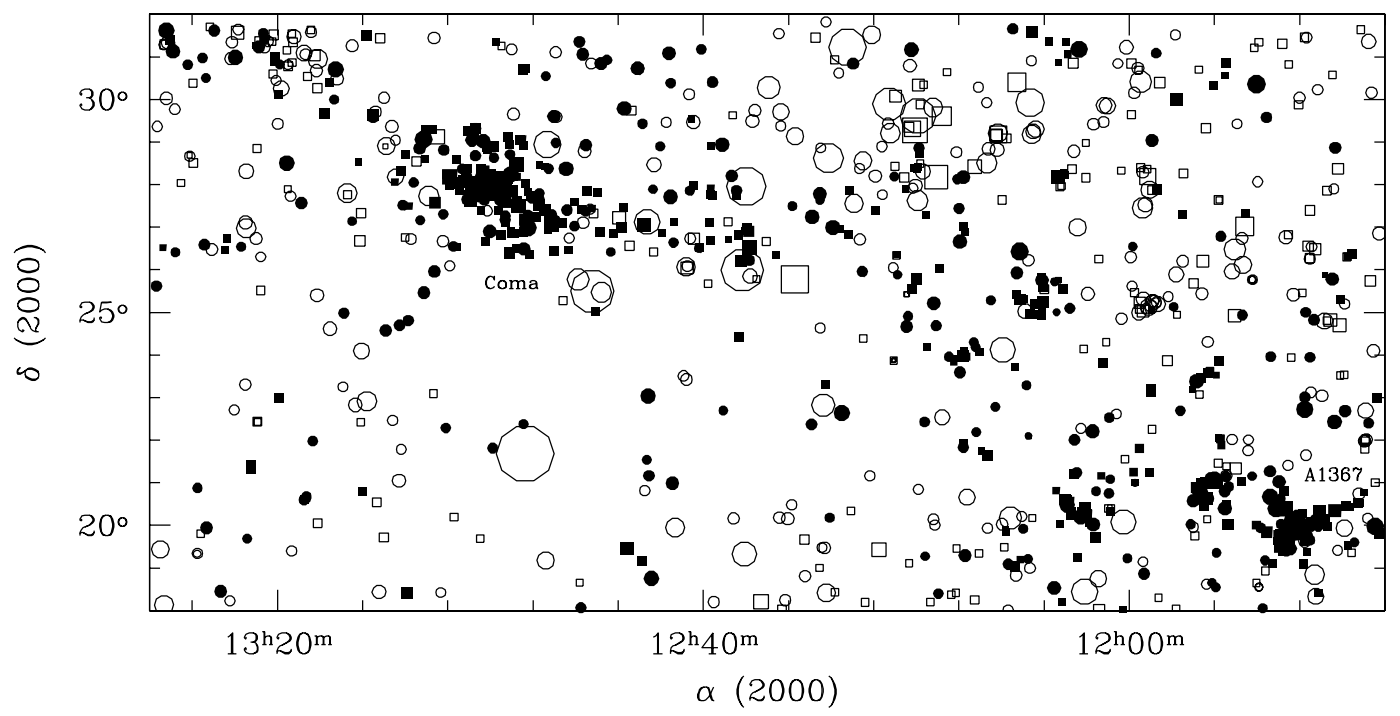

Fig. 2. Sky distribution of the 1127 CGCG galaxies in the Coma supercluster region. Spectroscopically confirmed supercluster members (filled symbols) are 649.

- Filter.

- Telescope.

- WCS parameters.

- Photometric effective zero point (when available) in mag/s for broad band images or in erg $\mathrm{cm}^{-2} \mathrm{~s}^{-1}$ for $\mathrm{H} \alpha$ images, such that $\log$ Flux $=Z p+\log C n t s-\log T_{\text {exp }}$. The effective zero point includes the atmospheric extinction term.

This homogenization is not yet complete. Its progress will be detailed in the online "GOLDMine news" section.

\section{Summary}

We undertook a 15 year research project focused on the properties of galaxies in the local universe which brought some new insight into their phenomenology and possibly will help reveal their origin and evolution (see Gavazzi 1993; Gavazzi \& Scodeggio 1996; Gavazzi et al. 1996, 2002; Boselli et al. 2001).

- A complete, optically selected sample of 3267 galaxies representative of all morphological types and luminosities was extensively surveyed through various observational windows from the $U V$ to the centimetric radio.

- The surveyed regions include the Virgo cluster and the Coma supercluster.
- Numeric parameters and images are made available to the international community through the World Wide Web site http://goldmine.mib.infn.it.

If your research benefits from the use of GOLDMine, we would appreciate the following acknowledgement in your paper: "This research has made use of the GOLDMine Database, operated by the Universita' degli Studi di Milano- Bicocca”. Please also cite the present paper.

Acknowledgements. We wish to thank all people who contributed with data to GOLDMine: Gery Bernstein, Christian Bonfanti, Barbara Catinella, Luis Carrasco, Fabienne Casoli, Alessandra Contursi, Luca Cortese, John Dickey, Anna Gallazzi, Bianca Garilli, Jorge IglesiasParamo, Walter Jaffe, Robert Kennicutt, James Lequeux, Silvia Martocchi, Paola Pedotti, Daniele Pierini, Isabella Randone, Gerry Sanvito, Ginevra Trinchieri, Richard Tuffs, Jose M. Vilchez, Stefano Zibetti. In particular the FOCA team (J. Donas, B. Milliard, M. Laget, M. Viton) is acknowledged for $U V$ fluxes made available prior to publication. We also wish to thank the Time Allocation Committee of many observatories where the data here collected were taken. GOLDMine is supported by the Physics Department of Università degli Studi di Milano-Bicocca. A.B. acknowledges financial support from the French Programme National Galaxies (PNG). P.F. acknowledges support from CNR/ASI grant I/R/27/00.

The GOLDMine web site has been created using the Python programming language, the Apache web server software and the MySQL 
database. We wish to thank the developers of these packages for freely distributing these resources that have made possible the construction of this site.

\section{References}

Binggeli, B., Sandage, A., \& Tammann, G. 1985, AJ, 90, 1681 Boselli, A., Gavazzi, G., Donas, J., \& Scodeggio, M. 2001, AJ, 121, 753

Dreyer, J. 1888, MMRAS, 49, 1 (NGC)

Dreyer, J. 1895, MMRAS, 51, 185 (IC)

Dreyer, J. 1908, MMRAS, 59, 105 (IC)

Gavazzi, G. 1993, ApJ, 419, 469

Gavazzi, G., \& Scodeggio, M. 1996, A\&A, 312, L29
Gavazzi, G., Pierini, D., \& Boselli, A. 1996, A\&A, 312, 397

Gavazzi, G., Carrasco, L., \& Galli, R. 1999a, A\&AS, 136, 227

Gavazzi, G., Boselli, A., Scodeggio, M., Belsole, E., \& Pierini, D. 1999b, MNRAS, 304, 595

Gavazzi, G., Franzetti, P., Scodeggio, M., Boselli, A., \& Pierini, D. 2000, A\&A, 361, 863

Gavazzi, G., Bonfanti, C., Sanvito, G., Boselli, A., \& Scodeggio, M. 2002, ApJ, 576, 135

Nilson, P. 1973, Uppsala General Catalogue of Galaxies, Uppsala, Obs. Ann. 6 (UGC)

Zwicky, F., Herzog, E., \& Wild, P. 1961-1968, Catalogue of Galaxies and of Clusters of Galaxies, Pasadena: California Institute Of Technology (CGCG) 